\title{
ARTICLE
}

Epidemiology

\section{Comparison of anal cancer screening strategies including standard anoscopy, anal cytology, and HPV genotyping in HIV-positive men who have sex with men}

\author{
Simon Pernot $\mathbb{D}^{1,2}$, Pauline Boucheron ${ }^{3}$, Hélène Péré ${ }^{2,4}$, Marie-Laure Lucas ${ }^{5}$, David Veyer ${ }^{2,4}$, Nadia Fathallah ${ }^{6}$, Vincent de Parades ${ }^{6}$, \\ Juliette Pavie ${ }^{5}$, Jeanne Netter ${ }^{1}$, Lio Collias ${ }^{5}$, Julien Taieb ${ }^{1,2}$, Sophie Grabar ${ }^{2,3}$ and Laurence Weiss ${ }^{2,5}$
}

BACKGROUND: There is no consensus on screening strategy of high-grade intraepithelial neoplasia (HGAIN). Guidelines range from clinical examination with digital anorectal examination followed by standard anoscopy (SA), to anal cytology (Pap) +/- HPV genotyping. We compared screening strategy yields based on Pap, SA, and HPV-16 genotyping alone or in combination in HIVMSM.

METHODS: Pap, SA, and HPV-16 genotyping were performed in all HIV-MSM attending a first anal cancer screening consultation in Paris, France. High-resolution anoscopy, the gold standard to detect HGAIN, was performed in the case of HPV-16 positivity or abnormal cytology. Yield was defined as the number of patients with HGAIN relative to the total number of patients screened. RESULTS: On 212 patients, the complete strategy (SA + Pap + HPV genotyping) yield (12.7\%) was significantly higher than that of SA $(3.3 \%, p<0.001)$ and HPV-16 alone $(6.6 \%, p<0.05)$. Although none of the other strategies were significantly different from the complete strategy, Pap + HPV-16 and Pap + SA had closer yields (about 11\%), with OR $=0.83(95 \% \mathrm{Cl}[0.44 ; 1.57])$ and $0.87(95 \% \mathrm{Cl}$ [0.46;1.64]), respectively.

CONCLUSIONS: Pap combined with HPV-16 genotyping or SA tended towards higher yields compared to Pap alone, and closer to that of the complete strategy.

British Journal of Cancer (2018) 119:381-386; https://doi.org/10.1038/s41416-018-0176-9

\section{INTRODUCTION}

The main identified risk factors for squamous cell carcinoma of the anus (SCCA) are human papilloma virus (HPV) infection, being a man who has sex with men (MSM), smoking, and human immunodeficiency virus (HIV) infection. ${ }^{1}$ MSM infected by HIV (HIV-MSM) are the highest risk group, with an 80-100 times higher risk of developing SCCA compared to the general population. ${ }^{2}$

The natural history of SCCA shares analogies with that of cervical cancer: a persistent infection by a high-risk HPV (HR-HPV), mainly HPV-16, ${ }^{3,4}$ the development of anal intraepithelial neoplasia (AIN) of low (LGAIN) and high grade (HGAIN) and potentially cancer. As for detection of cervical lesions, the Papanicolaou smear test (Pap) and HPV genotyping have been used in SCCA screening programs in HIV-MSM and have been suggested as effective to decrease SCCA incidence. ${ }^{5,6}$ However, there is no international consensus on SCCA screening. The most commonly accepted modality is anal $\mathrm{Pap}^{6-8}$ which is recommended by Palefsky et al. ${ }^{9}$ Pap alone or combined with digital anorectal examination (DARE) is currently applied in several guidelines in the US ${ }^{10-12}$ and Europe. ${ }^{13}$ However, anal Pap is of suboptimal sensitivity, which leads to underdiagnosis of lesions. HPV genotyping using polymerase chain reaction (PCR) has also been proposed, ${ }^{3,4,14}$ but was not included in guidelines because of its low specificity for the diagnosis of HGAIN. Some authors have proposed restricting genotyping to HPV-16; other HR-HPV such as HPV-18 may be considered as less relevant due to their lower prevalence in HIV-MSM and lower involvement in HGAIN. ${ }^{15,16}$ Following a positive anal Pap or HPV genotyping, high-resolution anoscopy (HRA), which is the gold standard for diagnosis of AIN, is recommended. Finally, clinical examination, based on a DARE and perianal skin examination at least with or without anoscopy, could detect some lesions. Although DARE alone is recommended in few guidelines, ${ }^{17}$ it is well established that it may detect early cancer but not HGAIN. ${ }^{18}$ DARE combined with a standard anoscopy (SA) is recommended in French guidelines for HIV-MSM. ${ }^{19}$ Even if SA diagnoses HGAIN in some cases, $^{20}$ it may underestimate this diagnosis and its performance as a screening tool has never been studied. All these recommendations are based on expert opinions,

\footnotetext{
${ }^{1}$ Department of Hepato-Gastroenterology and Digestive Oncology, Georges Pompidou European Hospital, APHP, Paris, France; ${ }^{2}$ Université Paris Descartes, Sorbonne Paris Cité, Paris, France; ${ }^{3}$ Department of Biostatistics and Epidemiology, Cochin-Hôtel Dieu Hospital, APHP, Paris, France; ${ }^{4}$ Department of Virology, Georges Pompidou European Hospital, APHP, Paris, France; ${ }^{5}$ Department of Clinical Immunology, Georges Pompidou European Hospital, APHP, Paris, France and ${ }^{6}$ Department of Proctology, Saint-Joseph Hospital, Paris, France

Correspondence: Simon Pernot (Simon.pernot@gmail.com) or Laurence Weiss (laurence.weiss@aphp.fr)

These authors contributed equally: Sophie Grabar, Laurence Weiss.

Presented in part at the 9th International AIDS Society conference; 24-26 July, 2017; Paris.
}

Received: 17 February 2018 Revised: 11 June 2018 Accepted: 18 June 2018

Published online: 20 July 2018 
as few data are available on the performances of global strategies, including anal Pap, HPV-16 genotyping, and SA each alone or combined in HIV-MSM. Consequently, the US Centers for Disease Control and Prevention (CDC) consider that while screening seems to be useful, more evidence is needed to determine the best screening methods. ${ }^{21}$

The aim of this study was to compare seven screening strategies to detect HGAIN in HIV-MSM, HGAIN definitely confirmed on a biopsy performed during HRA or SA: anal Pap, SA, and HPV-16 genotyping each alone, combination of two of them (Pap + SA, Pap + HPV-16 genotyping, or SA + HPV-16 genotyping), or combination of the three (complete strategy: SA + Pap + HPV-16 genotyping).

\section{PATIENTS AND METHODS}

Anal cancer screening program

An annual anal cancer screening program including Pap, SA, and HPV-16 genotyping was implemented for HIV-MSM followed up in the Department of Clinical Immunology of the Georges Pompidou European Hospital (Paris, France) in 2012. Consecutive patients were enrolled prospectively and had the three procedures referred thereafter as "complete strategy" (Pap, SA, and HPV16 genotyping). HRA was performed in the case of HPV-16 positivity or abnormal cytology (ASCUS, LSIL, HSIL). During SA, if AIN was suspected, a biopsy was performed (Fig. 1). The study was conducted in accordance with the Declaration of Helsinki. All participants received an information form and informed consent was collected. The use of their medical records for clinical research purposes was approved by the Commission Nationale de I'Informatique et des Libertés (CNIL Authorization No.: 1922081).

\section{Inclusion criteria}

All consecutive patients attending an anal cancer screening consultation for the first time between January 2012 and August 2016 were included in the study. Patients who underwent any prior anal screening test or who were referred with acute anal symptoms, or who had not followed the complete strategy (i.e., complete primary screening and then HRA within 6 months, if indicated) were excluded from the study.

\section{Screening and diagnostic procedures}

Anal Pap, HPV-16 genotyping, and SA were performed during the same consultation. All patients included in the study got the three screening tests.

Anal Pap. Anal specimen was collected with polyester fiber swab prior to any examination and lubrification. The swab was then processed using a liquid cytology technique prior to Papanicolaou staining, and finally analysed by a pathologist.

HPV genotyping. Anal specimens collected with a brush were removed on UTM medium. During the study, after DNA extraction, HPV-16 detection was performed using INNO-LiPA HPV Genotyping Extra (Innogenetics", Gent) from January 2012 to September 2014 and using a multiplex real-time PCR assay Anyplex ${ }^{\text {TM }}$ || HPV28 (Seegene , Seoul) from September 2014 to August 2016.

Standard anoscopy. One trained proctologist examined the anal margin and performed DARE and SA with the naked eye. In the event of any abnormality suggestive of AIN, a biopsy was performed. Clinical criteria for suspicion of HGAIN were not precisely defined, and included flat or slightly elevated condyloma, apparent vessels, imprecise borders, induration, or ulceration. HRA was performed in the case of positive screening. HRA is an examination of the squamocolumnar junction, anal canal, and perianal skin, under magnification using a colposcope, with application of $5 \%$ acetic acid solution and then Lugol's iodine.
Any suspicious lesion was biopsied under direct visualisation. All HRA were performed by a single trained proctologist who performs around 150 HRA a year, in respect of the IANS International Guidelines. ${ }^{22}$

Pathology. Centralised pathology examination was performed by the same experimented gastrointestinal pathologist. AIN were graded according to the Bethesda system, with AIN II and III being classified as HGAIN. ${ }^{23}$

\section{Statistical analysis}

Screening yield was defined as the number of patients with HGAIN relative to the total number of patients screened. Based on the tests results, we were able to know how many HGAIN would have been detected if only anal pap (pap alone) or only SA (SA alone) or HPV-16 genotyping (HPV-16 genotyping alone), or combination of screening tests (Pap + SA, etc.) would have been performed, defining the yield of each strategy. In the main analysis, each strategy yield was compared with that of the complete strategy (i.e., SA combined with anal Pap and HPV-16 genotyping), and anal Pap alone. Altogether, seven strategies were tested: each exam alone, all combinations of two exams in comparison to the complete strategy including the three screening methods. The odds ratio and 95\% confidence intervals were estimated.

All tests used were two-tailed and results were considered significantly different if $p$-value $<0.05$. All statistical analyses were performed using $\mathrm{R}$ software.

\section{RESULTS}

Study population

Of 253 HIV-MSM who attended a first SCCA screening consultation between 2012 and 2016, 212 (83.8\%) fulfilled the inclusion criteria and 41 were excluded, mostly because they did not attend the HRA within 6 months after the screening $(n=34)$. Patient characteristics are described in Table 1. Median age was 51 years and median duration of known HIV infection 15 years. CD4 cell count was above $500 / \mathrm{mm}^{3}$ in $73.8 \%$ of patients and HIV RNA was below 20 copies $/ \mathrm{mL}$ in $84 \%$. Baseline characteristics of excluded patients did not differ from those of the study population.

\section{Results of the screening strategies}

None of the three screening tests was positive in 126 patients (59.4\%). Pap was positive in 62 (29.2\%) patients, HPV-16 genotyping in $40(18.9 \%)$ patients, and SA in 19 (8.9\%) patients. Overall, $86(40.5 \%)$ patients had at least one positive test. Among them, 67 had no macroscopic suspicion of dysplasia and were referred for HRA. Finally, HGAIN was diagnosed by histology in 27 patients (12.7\%), including seven with macroscopic lesions at SA (Fig. 2).

The HGAIN detection yields of SA (3.3\%) and HPV-16 alone (6.6\%) were significantly lower than that of the complete strategy (12.7\%) (Table 2). When compared to the complete strategy, dual strategies including anal Pap + HPV-16 genotyping and anal Pap + SA had similar HGAIN detection yields (respectively $10.9 \%$, OR = $0.83[0.44 ; 1.57] p=0.65$ and $11.3 \%, \mathrm{OR}=0.87$ [0.46;1.64] $p=0.77$ ). Conversely, although not significantly different from the complete strategy, anal Pap alone and SA + HPV-16 genotyping had slightly lower HGAIN detection yield (both 9\%; 0.67 [0.34;1.30] $p=0.28$ ).

When strategies were compared to anal Pap alone, the detection yield with SA alone was significantly lower (respectively $3.3 \%$ vs $9 \%$ ). Although none of the yields of other strategies was significantly different from Pap alone, all bimodal strategies adding HPV-16 genotyping or SA to anal Pap, had a higher HGAIN detection yield than Pap alone, except for the combination of SA + HPV-16 genotyping. Finally, the yield with SA was significantly lower than the yields with all other strategies (alone or combined), except for HPV-16 genotyping (Supplementary Table 1). 


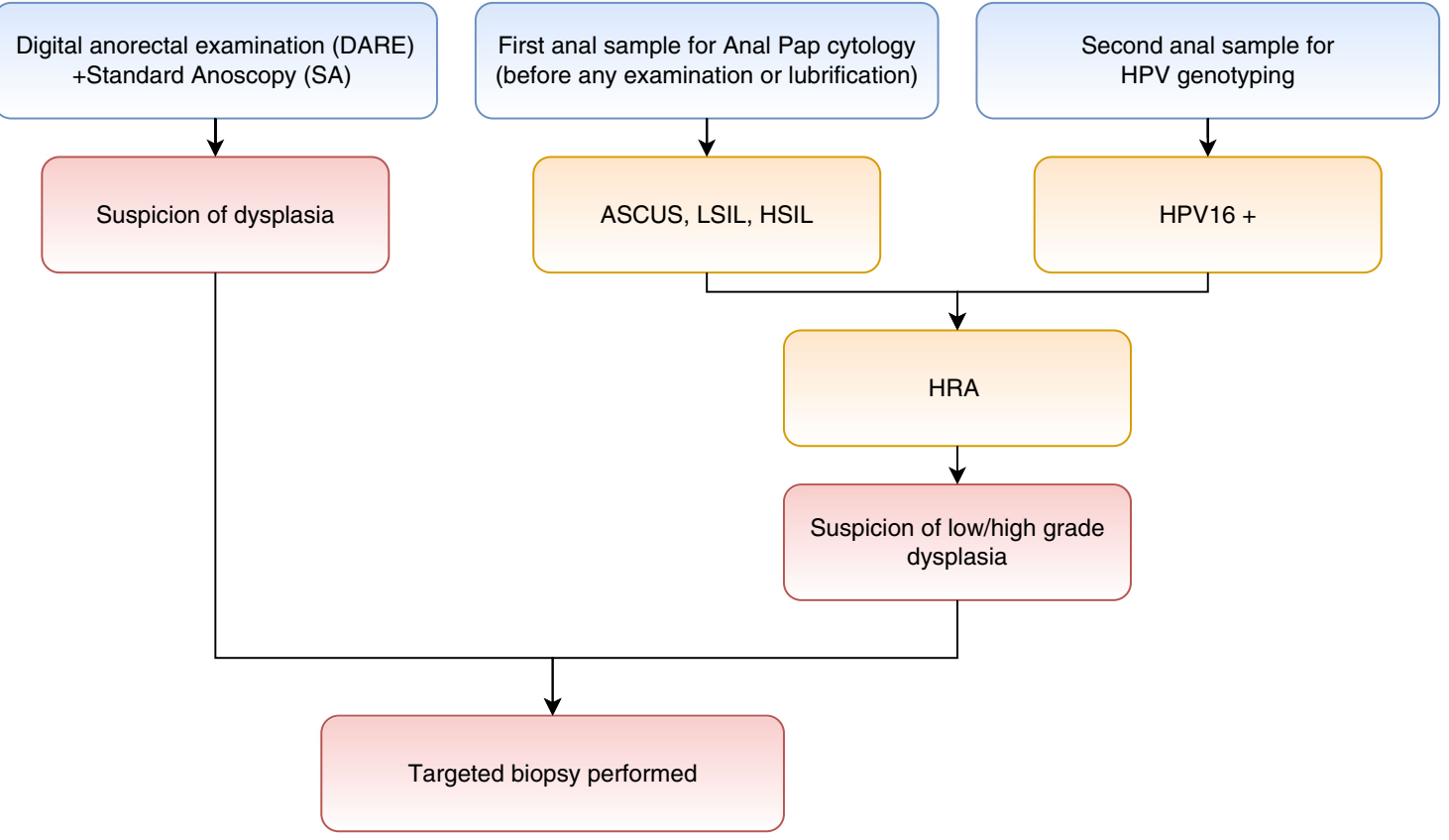

Fig. 1 Screening algorithm

Table 1. Patient characteristics ( $N=212$ HIV-MSM)

\begin{tabular}{ll}
\hline Patient characteristics & \\
\hline Age (years): median (IQR) & $51(45-57)$ \\
Time since HIV diagnosis (years): median (IQR) & $15.2(5.5-22.6)$ \\
Current antiretroviral treatment: yes $N(\%)$ & $209(98.6)$ \\
CD4 cell count $\left(/ \mathrm{mm}^{3}\right)$ : median (IQR) & $682(491-890)$ \\
CD4 nadir $\left(/ \mathrm{mm}^{3}\right):$ median (IQR) & $271(153-377)$ \\
HIV-RNA levels $<20$ copies/mL: $N(\%)$ & $173(84.0)$ \\
Smoking history & \\
Current smoker $N(\%)$ & $62(29.2)$ \\
Past smoker $N(\%)$ & $44(20.7)$ \\
No smoking history (\%) & $100(47.1)$ \\
ND (\%) & $6(28.3)$ \\
\hline
\end{tabular}

\section{DISCUSSION}

In the present study of HIV-MSM attending an anal cancer screening for the first time, the prevalence of HGAIN was $12.7 \%$. Among single screening modalities, anal Pap alone had the highest rate of HGAIN detection (9\%) compared to SA alone $(3.3 \%)$ or HPV-16 genotyping alone (6.6\%). However, Pap combined with HPV-16 genotyping or with SA gave a trend towards higher yields (about $11 \%$ ) in comparison to Pap alone or to SA+HPV-16 genotyping (9\%).

HRA is the gold standard for diagnosis of HGAIN. ${ }^{24}$ However, most authors agree that HRA cannot be used as a screening test ${ }^{5,9,14,22,25}$ because of its poor acceptability and accessibility. Thus, as in cervical pathology with colposcopy, HRA can only be used as a second-line diagnostic test for anal lesions after positive primary screening.

There is no international consensus on SCCA screening strategy. Pap is currently the most accepted screening test for HGAIN. The sensitivity of anal Pap ranges from 47 to $70 \%$ for the detection of AIN of any grade, but seems higher in HIV-MSM, ${ }^{7,26,27}$ reaching $89.2 \%$ for HGAIN detection in a recent study by Burgos et al. ${ }^{28}$ In our study, anal Pap alone detected only 19 of 27 (70\%) cases of
HGAIN, which is, however, more than SA or HPV16 genotyping alone (respectively, 7 and 14 cases).

For the detection of cervical cancers, the use of primary HPVbased screening rather than Pap is endorsed by several European guidelines, based on World Health Organization (WHO) recommendations. ${ }^{29}$ Its use with anal specimens has been proposed. ${ }^{14}$ In recent prospective studies, anal HPV genotyping had a sensitivity of more than $90 \%$ in HIV-infected and HIV-uninfected MSM when all high-risk HPV were considered. However, specificity was very low (15-25\%) because of the high prevalence of multiple HR-HPV infections in MSM. ${ }^{28,30}$ To increase HPV genotyping specificity, some authors have proposed restricting HRA referral to patients with positive HPV-16 only, based on the high involvement of HPV-16 in HGAIN and SCCA. ${ }^{15,31,32}$ In a multicentre study in HIV-infected men, the specificity of HPV-16 genotyping for the presence of HGAIN was $84 \%$, but its sensitivity was only $35 \%^{31}$. In the present study, HPV-16 was found in $40 / 212$ patients (18.9\%); 14 of them had HGAIN. If HPV-16 alone was considered, 13/27 HGAIN cases would not have been detected. HPV-16 genotyping alone was significantly inferior to the complete strategy $(\mathrm{OR}=$ $0.48 ;$ [0.23; 0.99]). Our data clearly indicate that HPV-16 genotyping should not be used as a screening method on its own. However, it would be interesting to consider each HR-HPV genotype alone or in combination as a way to improve the performance of HPV testing. Burgos et al. have shown that the sensitivity and specificity of detection of HPV-16 and/or 18 in predicting HGAIN were only $56 \%$ and $63 \%$, respectively. ${ }^{26}$ Other HR-HPV seem more prevalent than HPV-18 in HIV-MSM ${ }^{15,16}$ and it has recently been confirmed that being infected with two or more HR-HPV genotypes was correlated with HGAIN recurrence. ${ }^{33}$ This reinforces the potential interest of focusing on combinations of selected HR-HPV genotypes. Therefore, further investigations are needed to consider the implementation of HR-HPV genotypes other than HPV-16 in anal cancer screening.

In France, national guidelines recommend annual SA for SCCA screening in HIV-MSM. ${ }^{19}$ However, it is widely accepted that a clinical examination with SA including DARE, even performed by a specialist, may miss HGAIN because cases are mostly subclinical and detected only by HRA. In a study of 441 HIV-MSM, DARE failed to detect any of the 156 cases of HGAIN. ${ }^{18}$ In a prospective series 


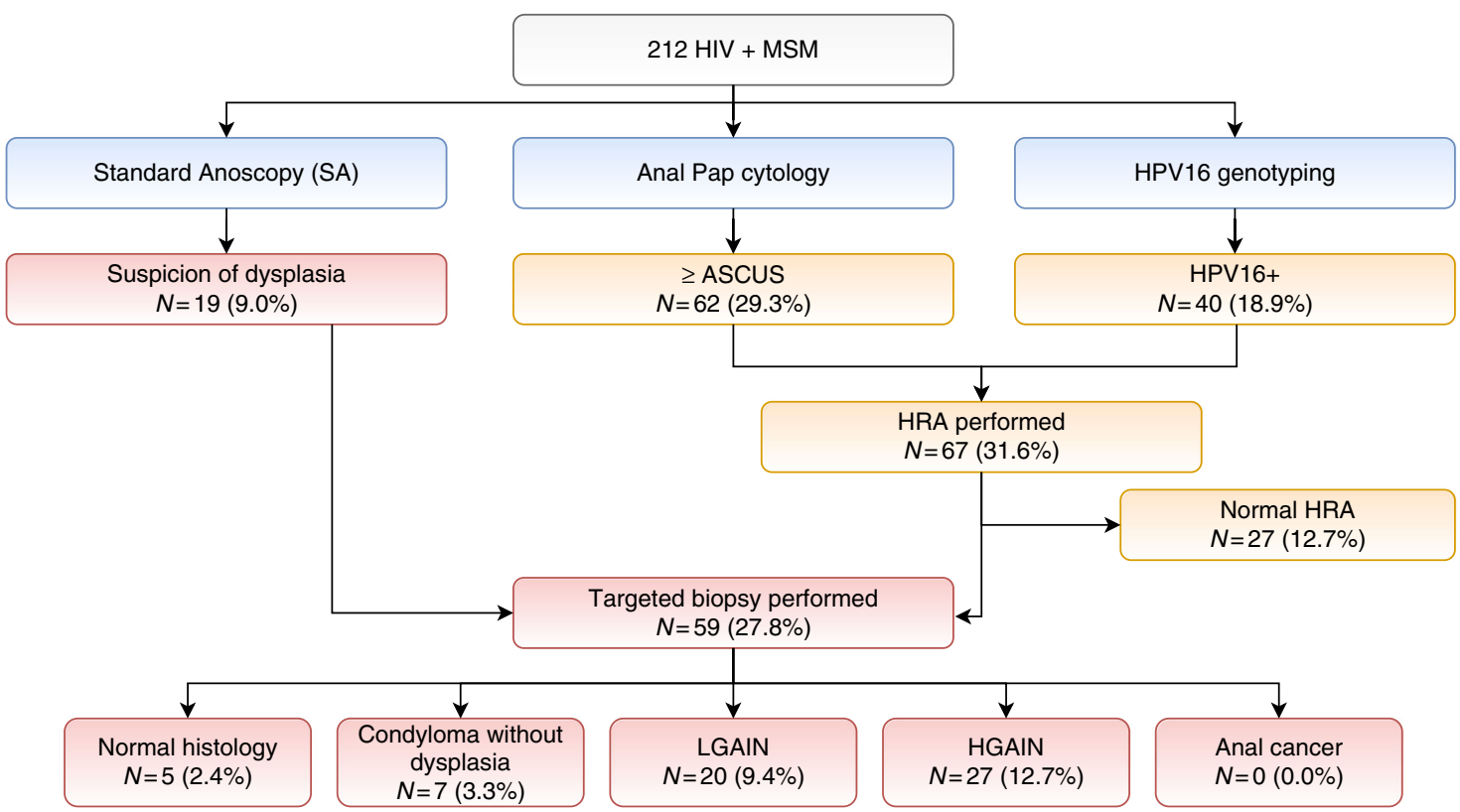

Fig. 2 Results of the complete strategy SA + Pap + HPV-16 genotyping

\begin{tabular}{|c|c|c|c|c|c|c|}
\hline HPV-16 genotyping & 40 & 39 & 26 & $\begin{array}{l}14 \\
(6.6 \%)\end{array}$ & $0.48[0.23 ; 0.99] p<0.05$ & $0.72[0.32 ; 1.56] p=0.47$ \\
\hline Pap & 62 & 59 & 40 & $\begin{array}{l}19 \\
(9.0 \%)\end{array}$ & $0.67[0.34 ; 1.30] p=0.27$ & Ref. \\
\hline Pap + HPV-16 genotyping & 75 & 75 & 48 & $\begin{array}{l}23 \\
(10.9 \%)\end{array}$ & $0.83[0.44 ; 1.57] p=0.65$ & $1.24[0.62 ; 2.48] p=0.63$ \\
\hline $\mathrm{SA}+\mathrm{Pap}$ & 70 & 51 & 52 & $\begin{array}{l}24 \\
(11.3 \%)\end{array}$ & $0.87[0.46 ; 1.64] p=0.77$ & $1.30[0.66 ; 2.59] p=0.52$ \\
\hline $\begin{array}{l}\text { SA + Pap + HPV-16 } \\
\text { genotyping }\end{array}$ & 86 & 67 & 59 & $\begin{array}{l}27 \\
(12.7 \%)\end{array}$ & Ref. & $1.48[0.76 ; 2.92] p=0.27$ \\
\hline
\end{tabular}

of 121 HIV-infected patients, SA was normal in $75 \%$ of cases of HGAIN. ${ }^{8}$ In our study, SA detected only 7 of 27 HGAIN cases (26\%).

Overall, considering our findings and the literature, anal Pap seems better than SA or HPV16 genotyping, but may be considered as suboptimal in detecting HGAIN if performed alone.

We found that adding SA to anal Pap slightly increased the rate of HGAIN detection (24/27 HGAIN, 89\% compared to 19/27). Considering that $S A$ is a simple tool, with a limited increase in costs, it may be implemented as part of the screening strategy. In a recent report by Burgos et al., HR-HPV genotyping was combined with anal cytology. This screening strategy identified almost all HGAIN lesions, but required HRA referral of almost $90 \%$ of patients, due to the lack of specificity of global HR-HPV testing. ${ }^{28}$ In our study, compared to Pap alone, there was a trend towards a higher HGAIN detection rate when combining Pap with HPV-16 genotyping (23/27 HGAIN, 85\%) and only 75/212 (35\%) patients would have required HRA.

Acceptability by the patient and the physician is a major concern for the success of a screening program. We and others have shown that more than $50 \%$ of HIV-MSM had never undergone any anal screening test, despite recommendations. Among explanations, lack of information by physicians and lack of time/motivation were highlighted. ${ }^{34}$ Therefore, it is likely that a screening strategy allows self-sampling would be more acceptable. Anal Pap self-sampling has been shown to be acceptable and efficient as a screening strategy, ${ }^{35}$ whereas HPV genotyping self-sampling is currently being studied. ${ }^{36}$

Finally, the interest of screening HGAIN is still unresolved as it has never been shown that such screening could lead to a decrease in cancer incidence, morbidity, or mortality. The natural history of anal cancer is still an unresolved issue. Progression from HGAIN to cancer is not known, and probably lower than in cervical neoplasia. Based on a meta-analysis, Machalek et al. report a theoretical rate of progression of high grade anal dysplastic lesions (or HSIL) of 1/377 (0.27\%) among HIV-MSM and 1/4 196 $(0.024 \%)$ among HIV-uninfected MSM. ${ }^{37}$ Moreover, regression of HGAIN have been described either spontaneously or with immune restoration under ART. ${ }^{38,39}$ 
There is a need for prospective longitudinal and intervention studies to assess the interest of treating HGAIN in preventing the occurrence of cancer. Indeed, studies evaluating therapeutic intervention on HGAIN were mostly retrospective, not controlled, or too small. ${ }^{40}$ Only one small randomised trial had tested Imiquimod versus placebo in 64 patients. ${ }^{41}$ However, the primary outcome in this study was the regression of HGAIN; progression to cancer was not assessed. Nevertheless, as for detection of cervical lesions, screening programs in HIV-MSM have been suggested as being effective to decrease SCCA incidence. ${ }^{5,6}$ The ongoing ANCHOR study (NCT02135419), currently recruiting more than 5000 HIV patients with HGAIN, randomised into two arms: experimental arm (topical or ablative treatment of lesions) or monitoring every 6 months, will provide a better understanding of the natural history of HGAIN and relevance of treatment.

Our study had some limitations. Firstly, it was not designed to assess the sensitivity and specificity of each strategy, as HRA was only performed in patients with a positive screening test. Secondly, $16.2 \%$ of patients $(N=41 / 253)$ were excluded from the study because they did not adhere to the complete screening strategy. Thirdly, we found a lower prevalence of HGAIN than the $30-42 \%$ reported. ${ }^{31,42}$ The prevalence may have been underestimated because of exclusion of patients with symptoms or with any history of previous screening and/or by the design of the study. And we cannot exclude that some patients with negative screening tests had HGAIN, as HRA was not performed in all patients. However, the low prevalence is consistent with the prevalence observed in other recent French cohorts ${ }^{39,43}$ and mirrors a trend in HGAIN epidemiology in France. This low prevalence and the relatively small study population may have underpowered the study. Finally, ours was a single-centre study and the reproducibility and external validity of our results should be confirmed.

In conclusion, among the single screening strategies, anal Pap alone had a higher HGAIN detection yield than SA and HPV-16 genotyping. Among the dual combination strategies, anal Pap + HPV-16 and SA + anal Pap had detection yields similar to that of the complete strategy. However, even though SA decreased the number of HRA performed, it is likely that it might also affect participation or acceptance. In the perspective of self-sampling, anal Pap + HPV-16 genotyping might be the best strategy to increase screening acceptance and to identify HGAIN in HIV-MSM, although it would increase the need for HRA. However, the added value of screening using detection of HPV-16 combined with other selected HR-HPV remains to be assessed in further studies. In addition, extending HRA accessibility is a remaining challenge that needs to be addressed. Finally, these strategies should be evaluated in further studies in the context of low HRA accessibility and health economics systems, with regard to acceptability by the patients.

\section{AUTHOR CONTRIBUTIONS}

Study design: Simon Pernot, Sophie Grabar, Laurence Weiss. Data acquisition: Simon Pernot, Marie-Laure Lucas, Juliette Pavie, Hélène Peéé, David Veyer, Julien Taieb, Nadia Fathallah, Vincent de Parades, Juliette Pavie, Jeanne Netter, Lio Collias, Laurence Weiss. Quality control of data: Simon Pernot, Pauline Boucheron, Sophie Grabar. Data analysis and interpretation: Simon Pernot, Pauline Boucheron, Sophie Grabar, Laurence Weiss, Julien Taieb, Hélène Péré. Statistical analysis: Pauline Boucheron, Sophie Grabar. Manuscript preparation and editing: Simon Pernot, Pauline Boucheron, Sophie Grabar, Laurence Weiss. Manuscript review: All authors.

\section{ADDITIONAL INFORMATION}

Supplementary information is available for this paper at https://doi.org/10.1038/ s41416-018-0176-9.

Competing interests: The authors declare no competing interests.
Availability of data and material: Data are available at the Department of Biostatistics and Epidemiology, Cochin-Hôtel Dieu Hospital, APHP, Paris, France.

Ethics approval and consent to participate: The study was conducted in accordance with the Declaration of Helsinki. All participating patients received a written information note and gave their consent for the study and allowed the use of their medical records for clinical research purposes. The study was approved by the Commission Nationale de I'Informatique et des Libertés (CNIL Authorization No.: 1922081).

Note: This work is published under the standard license to publish agreement. After 12 months the work will become freely available and the license terms will switch to a Creative Commons Attribution 4.0 International (CC BY 4.0).

\section{REFERENCES}

1. Medford, R. J. \& Salit, I. E. Anal cancer and intraepithelial neoplasia: epidemiology, screening and prevention of a sexually transmitted disease. Can. Med. Assoc. J. 187, 111-115 (2015).

2. Piketty, C. et al. Incidence of HIV-related anal cancer remains increased despite long-term combined antiretroviral treatment: results from the french hospital database on HIV. J. Clin. Oncol. 30, 4360-4366 (2012).

3. Hoots, B. E., Palefsky, J. M., Pimenta, J. M. \& Smith, J. S. Human papillomavirus type distribution in anal cancer and anal intraepithelial lesions. Int. J. Cancer 124, 2375-2383 (2009).

4. De Vuyst, H., Clifford, G. M., Nascimento, M. C., Madeleine, M. M. \& Franceschi, S. Prevalence and type distribution of human papillomavirus in carcinoma and intraepithelial neoplasia of the vulva, vagina and anus: a meta-analysis. Int. J. Cancer 124, 1626-1636 (2009).

5. Blaser, N. et al. Impact of screening and ART on anal cancer incidence in HIVpositive men who have sex with men: mathematical modeling study. AIDS. 31, 1859-1866 (2017).

6. Berry, J. M. et al. Progression of anal high-grade squamous intraepithelial lesions to invasive anal cancer among HIV-infected men who have sex with men. Int. J. Cancer 134, 1147-1155 (2014).

7. Nathan, M. et al. Performance of anal cytology in a clinical setting when measured against histology and high-resolution anoscopy findings. AIDS 24, 373-379 (2010).

8. Berkelmans, I. et al. Faut-il préférer la cytologie anale plutôt qu'un examen anuscopique dans le cadre du dépistage de la dysplasie anale chez le VIH? (Paris, 2012).

9. Park, I. U. \& Palefsky, J. M. Evaluation and management of anal intraepithelial neoplasia in HIV-negative and HIV-positive men who have sex with men. Curr. Infect. Dis. Rep. 12, 126-133 (2010).

10. Kaplan, J. E., Masur, H. \& Holmes, K. K., USPHS, Infectious Disease Society of America. Guidelines for preventing opportunistic infections among HIVinfected persons-2002. Recommendations of the U.S. Public Health Service and the Infectious Diseases Society of America. MMWR Recomm. Rep. 51, 1-52 (2002).

11. Aberg, J. A. et al. Primary care guidelines for the management of persons infected with HIV: 2013 update by the HIV Medicine Association of the Infectious Diseases Society of America. Clin. Infect. Dis. 58, 1-10 (2014).

12. Anal Dysplasia and Cancer Guideline-AIDS Institute Clinical Guidelines [Internet]. [cité 7 déc 2017]. Disponible sur: http://www.hivguidelines.org/adult-hiv/ preventive-care-screening/anal-dysplasia-cancer/.

13. EACS Guidelines [Internet]. [cité 21 juin 2017]. Disponible sur: http://www. eacsociety.org/guidelines/eacs-guidelines/eacs-guidelines.html.

14. Goldstone, S. E. \& Moshier, E. Detection of oncogenic human papillomavirus impacts anal screening guidelines in men who have sex with men. Dis. Colon Rectum 53, 1135-1142 (2010).

15. Sahasrabuddhe, V. V. et al. Human papillomavirus genotype attribution and estimation of preventable fraction of anal intraepithelial neoplasia cases among HIV-infected men who have sex with men. J. Infect. Dis. 207, 392-401 (2013).

16. Darwich, L. et al. Distribution of human papillomavirus genotypes in anal cytological and histological specimens from HIV-infected men who have sex with men and men who have sex with women. Dis. Colon Rectum 56, 1043-1052 (2013).

17. Ong, J. J., Chen, M., Grulich, A. E. \& Fairley, C. K. Regional and national guideline recommendations for digital ano-rectal examination as a means for anal cancer screening in HIV positive men who have sex with men: a systematic review. BMC Cancer 14, 557 (2014). 
18. Kreuter, A. et al. Anal carcinoma in human immunodeficiency virus-positive men: results of a prospective study from Germany. Br. J. Dermatol. 162, 1269-1277 (2010).

19. Prise en charge médicale des personnes vivant avec le VIH: Rapport 2013Recommandations du groupe d'experts [Internet]. Disponible sur: http:// solidarites-sante.gouv.fr/IMG/pdf/Rapport_Morlat_2013_Mise_en_ligne-6.pdf.

20. Abramowitz, L. et al. Determinants of macroscopic anal cancer and precancerous lesions in 1206 HIV-infected screened patients. Colorectal Dis. 18, 997-1004 (2016).

21. Sexually Transmitted Diseases Treatment Guidelines, 2015 [Internet]. [cité 21 juin 2017]. Disponible sur: https://www.cdc.gov/mmwr/preview/mmwrhtml/rr6403a1. htm.

22. Hillman, R. J. et al. 2016 IANS international guidelines for practice standards in the detection of anal cancer precursors. J. Low Genit. Tract Dis. 20, 283-291 (2016).

23. Darragh, T. M. et al. The Lower Anogenital Squamous Terminology Standardization project for HPV-associated lesions: background and consensus recommendations from the College of American Pathologists and the American Society for Colposcopy and Cervical Pathology. Int. J. Gynecol. Pathol. 32, 76-115 (2013).

24. Camus, M. et al. Which lesions should be biopsied during high-resolution anoscopy? Prospective descriptive study of simple morphological criteria. J. Low Genit. Tract Dis. 19, 156-160 (2015).

25. Chiao, E. Y., Giordano, T. P., Palefsky, J. M., Tyring, S. \& El Serag, H. Screening HIVinfected individuals for anal cancer precursor lesions: a systematic review. Clin. Infect. Dis. 43, 223-233 (2006).

26. Palefsky, J. M. et al. Anal cytology as a screening tool for anal squamous intraepithelial lesions. J. Acquir. Immune Defic. Syndr. Hum. Retrovirol. 14, 415-422 (1997).

27. Berry, J. M. et al. Performance characteristics of anal cytology and human papillomavirus testing in patients with high-resolution anoscopy-guided biopsy of high-grade anal intraepithelial neoplasia. Dis. Colon Rectum 52, 239-247 (2009).

28. Burgos, J. et al. The role of oncogenic HPV determination for diagnosis of highgrade anal intraepithelial neoplasia in HIV-infected MSM. AIDS 31, 2227-2233 (2017).

29. Wentzensen, N. et al. Eurogin 2016 Roadmap: how HPV knowledge is changing screening practice. Int. J. Cancer 140, 2192-2200 (2017)

30. Schofield, A. M. et al. A prospective study of anal cancer screening in HIV-positive and negative MSM. AIDS 30, 1375-1383 (2016)

31. Wilkin, T. et al. High-grade anal intraepithelial neoplasia among HIV-1-infected men screening for a multicenter clinical trial of a human papillomavirus vaccine. HIV Clin. Trials 14, 75-79 (2013).
32. Steinau, M. et al. Human papillomavirus prevalence in invasive anal cancers in the United States before vaccine introduction. J. Low Genit. Tract Dis. 17, 397-403 (2013).

33. Burgos, J. et al. Risk factors of high-grade anal intraepithelial neoplasia recurrence in HIV-infected MSM. AIDS 31, 1245-1252 (2017).

34. Vanhaesebrouck, A. et al. HIV1-positive men who have sex with men (HIV1MSM) knowledge and attitudes towards anal cancer screening: a crosssectional study. Ann. Oncol. 27, https://doi.org/10.1093/annonc/mdw385.03 (2016).

35. Chin-Hong, P. V. et al. Comparison of patient- and clinician-collected anal cytology samples to screen for human papillomavirus-associated anal intraepithelial neoplasia in men who have sex with men. Ann. Intern. Med. 149, 300-306 (2008)

36. Tamalet, C. et al. Feasibility and acceptability of anal self-sampling for human papillomavirus screening in HIV-infected patients. Intervirology 59, 118-122 (2016).

37. Machalek, D. A. et al. Anal human papillomavirus infection and associated neoplastic lesions in men who have sex with men: a systematic review and metaanalysis. Lancet Oncol. 13, 487-500 (2012).

38. Tong, W. W. Y. et al. Progression to and spontaneous regression of high-grade anal squamous intraepithelial lesions in HIV-infected and uninfected men. AIDS 27, 2233-2243 (2013).

39. Piketty, C. et al. Lack of regression of anal squamous intraepithelial lesions despite immune restoration under CART. AIDS 27, 401-406 (2013).

40. Macaya, A., Muñoz-Santos, C., Balaguer, A. \& Barberà, M. J. Interventions for anal canal intraepithelial neoplasia. Cochrane Database Syst. Rev. 12, CD009244 (2012).

41. Fox, P. A et al. A double-blind, randomized controlled trial of the use of imiquimod cream for the treatment of anal canal high-grade anal intraepithelial neoplasia in HIV-positive MSM on HAART, with long-term follow-up data including the use of open-label imiquimod. AIDS 24, 2331-2335 (2010).

42. Gaisa, M., Sigel, K., Hand, J. \& Goldstone, S. High rates of anal dysplasia in HIVinfected men who have sex with men, women, and heterosexual men. AIDS 28, 215-222 (2014)

43. Etienney, I. et al. Dépistage des lésions anales précancéreuses; Que sommes-nous capables de diagnostiquer?médicale française d'hépato-gastroentérologie et d'oncologie digestive [Internet]. [cité 9 oct 2017]. Disponible sur: http://www. snfge.org/content/depistage-des-lesions-anales-precancereuses-qu. 